\title{
Elmodelo español de deporte en la universidad: fundamentación, descripcióny orientaciones para su gestión ética \\ The Spanish model of sport at university: principles, description and guidelines for its ethical management
}

\author{
Óscar Chiva Bartoll, Carlos Hernando Domingo \\ Universitat Jaume I de Castellón
}

\begin{abstract}
Resumen: El presente artículo analiza los fundamentos del actual modelo español de deporte en la universidad, con el objeto de orientar la oferta de los servicios de deporte universitarios. En primer lugar se propone una reflexión sobre el hecho deportivo contemporáneo ya que, como se verá, de él emanan algunas modalidades susceptibles de ser introducidas en el marco universitario, aunque también se da cuenta de algunas vertientes que no gozarían de la pretendida legitimidad. Posteriormente, para discernir sobre la adecuación o no de esos modelos de práctica el artículo delimita el sentido o razón de ser del deporte en la universidad, defendiendo que su misión debe estar perfectamente alineada con las metas de la institución universitaria. En esa línea se argumenta que el bien interno de los servicios de deporte universitarios radica necesariamente en promover una oferta deportiva proclive a mejorar la educación y el desarrollo social. Finalmente, a partir de los argumentos esgrimidos y aprovechando las orientaciones del Informe del Deporte en la Universidad del Plan Integral para la Actividad Física y el Deporte (Consejo Superior de Deportes, 2010), se ofrecen algunas claves para determinar un horizonte deseable de gestión ética.

Palabras clave: deporte en la universidad, modelo deportivo, gestión deportiva, gestión ética, servicio de deportes.
\end{abstract}

\begin{abstract}
This paper analyzes the principles of the current model of Spanish sport in college, in order to guide the supply of college sport departments. First, we propose a reflection on the concept of contemporary sport as a social fact. This review shows some sport approaches that could be introduced in college sport departments. Nevertheless, it also shows that some conceptions would not be legitimate. Consequently, the paper defines the meaning of sport at university and reflects on the adequacy of the analyzed sport conceptions. We argue that its mission must be perfectly aligned with the goals of the university. According to the stated arguments, we affirm that the inner sense of university sports departments lies in promoting sporting activities aiming to improve education and social development. Finally, based on the arguments exposed and taking into account the orientations of the Report of Sport at University, which belongs to the Comprehensive Plan for Physical Activity and Sport (Consejo Superior de Deportes, 2010), we offer some clues to determine a desirable horizon of ethical management.
\end{abstract}

Key words: sport in college, sports model, sports management, ethical management, sports department.

\section{Introducción}

El deporte en la universidad ha sufrido una intensa evolución desde sus inicios. Sólo en las últimas décadas del siglo XX ha pasado de una situación de fuerte centralización basada en un enfoque competitivo, a funcionar últimamente en contextos de mayor autonomía universitaria y con un enfoque bastante más amplio (López-Yeste, 1999). Hoy en día, a raíz de su faceta educativa y formadora, la oferta de actividad físico-deportiva constituye un servicio que la sociedad exige de pleno derecho a las diferentes universidades. Para dar respuesta a esta demanda, la institución universitaria ha ido implantando progresivamente una serie de estructuras de gestión específicas, conocidas generalmente como unidades o servicios de deporte universitarios (Almorza, Yébenes, Bablé, Rivas, Ronquete \& Casadi, 2011).

A partir de las diferentes vías de evolución que en los últimos tiempos ha sufrido el hecho deportivo, los modelos de práctica físicodeportiva que ofertan las universidades presentan diferencias significativas entre sí. Por ello, para determinar con claridad el bien interno de los servicios de deporte universitarios, es necesaria una reflexión sobre las características particulares de su praxis.

El objetivo que aquí nos proponemos es indagar sobre los fundamentos del modelo español de deporte en la universidad, desvelando el bien interno desde el que se construye la legitimidad de su praxis, para estar finalmente en disposición de orientar y guiar su gestión. Por lo que más allá de abordar una investigación basada en desvelar datos objetivos de su praxis, aquí se desarrolla un trabajo filosófico encaminado a desvelar una aproximación deontológica basada en lo que debe ser dicha praxis. Es decir, se aspira a conocerlo y describirlo para dar un paso más allá: orientarlo y guiarlo.

Fecha recepción: 03-12-13- Fecha envío revisores: 08-12-13- Fecha de aceptación: 16-02-14 Óscar, Chiva Bartoll

Av de Vicent Sos Baynat, s/n,

12071 Castellón de la Plana, Castellón

ochiva@uji.es
Esta iniciativa sellevaráa cabo desdeuna aproximación metodológica hermenéutico-crítica, que permitirá establecer la hoja de ruta a seguir. El método hermenéutico-crítico, de amplia tradición filosófica, trata de averiguar desde el interior de cada actividad los fines que le dan sentido y la legitiman socialmente. Una vez descubierto el bien interno de la actividad, este método propone cultivar tanto los hábitos y valores de la praxis, como los medios y procedimientos que pueden ayudar a alcanzar dicho fin (Conill, 2006; Cortina, 1993).

Este enfoque metodológico propone además una estructura de carácter circular compuesta por varias fases (Cortina, 1993), con las que se ha hecho concordar el plan de trabajo establecido. El artículo se organiza en torno a cuatro apartados. El primero de ellos aborda una delimitación del deporte actual desde su faceta como dimensión social, es decir, como hecho deportivo. En su interior se recogen algunas de las clasificaciones más generalizadas del hecho deportivo contemporáneo, de forma que quedan reflejadas las diferentes tendencias susceptibles de ser promovidas por los servicios de deporte universitarios. En el segundo apartado se hace una aproximación al modelo de práctica físicodeportiva que corresponde exigir a las universidades. Este apartado da voz tanto a las principales referencias legales, como a algunos especialistas reconocidos en la materia, defendiendo con ello el perfil educativo y democratizador del enfoque requerido. El tercer apartado clarifica qué vertientes del hecho deportivo contemporáneo encuentran legitimidad en la oferta universitaria y cuáles no. Por último, en el cuarto apartado se ofrecen propuestas de gestión que podrían facilitar el acercamiento hacia ese horizonte. Esta orientación engloba dos niveles de organización del deporte en la universidad: uno basado en la idea de deporte en la universidad como institución, y otro basado en la gestión de los propios servicios de deporte en tanto que entidades autónomas. Anivel institucional la sugerencia se apoyará en el Informe de Deporte en la Universidad del Plan Integral para la Actividad Física y el Deporte (Consejo Superior de Deportes, 2010). A nivel organizacional, a saber, centrando la mirada en los propios servicios de deporte, se plantearán sugerencias de mejora relativas a dos dimensiones inseparables de la gestión: la ética y la estratégica. 


\section{El hecho deportivo contemporáneo}

Para entender los distintos enfoques que la práctica físico-deportiva ha llegado a adquirir en la universidad, conviene hacer una aproximación previa al estado del hecho deportivo en general; ya que se trata de un concepto que tras su aparente simplicidad esconde una enorme complejidad social y cultural (García-Ferrando, 1990, 2006; GarcíaFerrando \& LLopis, 2011), que queda reflejada también en la vertiente universitaria.

La dificultad de delimitar el término deporte radica en que no puede darse una definición que abarque unívocamente todo su significado. Tanto es así que reconocidos autores como Isidori (2011) y Parlebás (2001), debido a esa falta de acuerdo y consenso, llegan a hablar de un problema de indefinición. En definitiva, puede decirse que existen tantos factores implicados en la acción deportiva, que el deporte debe ser tratado como un fenómeno multidimensional (Almeida, 2001).

En relación a las pretensiones del presente artículo, nos apoyamos en Cagigal (1996)cuando aludea la existencia de dos caras específicamente independientes del fenómeno deportivo: una como realidad ontológica y otra, derivada de ésta, como realidad social. Como realidad ontológica el deporte es algo que existe de un modo intrínseco a la naturaleza humana y que, por tanto, se ha manifestado siempre donde el ser humano ha existido. Ahora bien, a partir de esa realidad ontológica emerge un entramado de relaciones intersubjetivas que convierten al deporte en una manifestación social. Aesta aproximación del deporte la denominaremos en adelante hecho deportivo (Domínguez, 1995).

Una vez aclarada la naturaleza social de nuestra aproximación, que en definitiva es la que se refleja en el marco universitario, veremos cuál es la situación del hecho deportivo contemporáneo. A diferencia de las manifestaciones físico-deportivas de épocas anteriores, para autores como Vicente (2011), el deporte contemporáneo incluye una serie de rasgos distintivos propios de las sociedades actuales. En esta misma línea Cagigal (1996) percibe que el deporte posterior a los años 70 dista mucho del que inventaron los ingleses. En la época contemporánea emergen otros roles, estructuras y valores como el espectáculo, la política, la técnica, la ciencia, el profesionalismo, la globalización, etc., (Miller, Lawrence, McKay \& Rowe, 2001). Bajo estos preceptos, el deporte asume un papel social importante en el momento histórico actual. Además, de la mano de Mechikoff y Estes (2005), se puede afirmar que hoy en día existen más posibilidades que nunca de participar en multitud de modalidades y variantes de práctica deportiva y actividad física.

En relación a esa amplitud de posibilidades, se han propuesto diferentes modelos y clasificaciones entre las que se presentan a continuación algunas de las más destacadas. Por ejemplo Cagigal (1996) indica: «La primera y fundamental clasificación que se impone, partiendo de la misma naturaleza del hecho deportivo es: el deporte como $<<$ praxis >>; y el deporte como espectáculo» (p. 765).

En la misma línea, Rodríguez (2008) explica que a día de hoy existen dos corrientes divergentes que, condicionadas por diferentes motivaciones, desempeñan funciones distintas en la sociedad: el deporte-espectáculo y el otro deporte. Por una parte estaría el deporte-espectáculo, promovido por intereses económicos y comerciales, y con frecuencia vinculado a la profesionalización. Y, por otra parte, lo que élllama el otro deporte, refiriéndose a aquel que queda ajeno a la propaganda, la comercialización, la instrumentalización política, etc.

García-Ferrando (1990) diferencia por ejemplo los deportes en función de la presencia del carácter utilitario del juego, destacando desde este enfoque un continuo en el que se encontrarían las siguientes perspectivas: deportes formales, deportes semiformales y deportes informales. En esta línea, se aclara que la principal tendencia del deporte contemporáneo detectada por los sociólogos es la que relaciona al ocio con el deporte (García-Ferrando, 1990; Puig \& Monteagudo, 2004). Tanto es así que ante la concepción del deporte como actividad de tiempo libre, una de las preferencias de la población respecto a la dedicación de su tiempo de ocio recae en el fenómeno deportivo.

Por otra parte, López-Yeste (1999) aporta una nueva referencia atendiendo a este carácter bipolar entre la competición y la recreación, desde el que se diferencian claramente los modelos de deporte actuales. Siguiendo las orientaciones planteadas por esta autora, conviene tener en cuenta que de cada una de estas perspectivas emanan una serie de valores diferentes, lo cual debe ser tenido en cuenta al establecer el modelo de práctica físico-deportiva en la universidad.

El conjunto de clasificaciones recogidas hasta el momento ofrecen una idea clara de los distintos derroteros que ha tomado el hecho deportivo actual. A riesgo de ser excesivamente sintéticos, podemos afirmar que las distintas clasificaciones responden a dos criterios básicos: (1) quien practica y (2) con qué objetivo. Para apreciar esta cuestión con mayor claridad se presenta la tabla 1 en la que se recogen las clasificaciones explicadas.

\begin{tabular}{ccc}
\multicolumn{2}{l}{ Tabla 1. Compilación de clasificaciones de modelos de deporte contemporáneos. (Elaboración propia) } \\
\hline Autores & Modelos dedeporte \\
\hline Cagigal (1996) & Deporte praxis & Deporte como espectáculo \\
Rodríguez (2008) & Otro deporte & Deporte Espectáculo \\
García-Ferrando (1990) & Deportes informales & Deportes semi formales Deportes formales \\
García-Ferrando (1990) & Deporte para todos & Deporte Espectáculo \\
López-Yeste (1999) & Deportede recreación & Deporte de compet ición \\
\hline
\end{tabular}

Esta revisión panorámica muestra los principales modelos que configuran la realidad del hecho deportivo actual. Sin embargo, a las clasificaciones recogidas creemos oportuno sumar una nueva propuesta en la que cabría contemplar tres modelos de deporte: el deporte práctica o deporte para todos por una parte; el deporte rendimiento, deporte de élite o deporte competición por otra parte; y el deporte espectáculo o deportenegocio.

El deporte práctica o deporte para todos recogería aquel modelo de actividad física en el que los practicantes son individuos que, independientemente de su edad o condición, practican libremente movidos por el objetivo de alcanzar mejores cotas de bienestar, salud, recreación, autoestima, superación personal, etc.

El deporte rendimiento, deporte de élite o deporte de competición albergaría a aquellos participantes que persiguen la consecución de un elevado nivel de rendimiento deportivo, centrando el objetivo de su práctica en obtener los mejores resultados posibles en las competiciones regladas y regidas por las federaciones e instituciones correspondientes.

Y finalmente, el deporte espectáculo o deporte negocio sería aquel que se vale del deporte de rendimiento, élite o competición para alcanzar un objetivo económico a través de diferentes vías de explotación. Este modelo aprovecha y entiende la espectacularidad y el valor estético y agonístico de la práctica deportiva como un producto de mercado con el que obtener ganancias. En este punto, puede afirmarse con Elías y Dunning (1986) que el deporte basado en el ocio se convierte en un negocio, dando pie también a la profesionalización de los deportistas.

En definitiva, entendemos que los diferentes modelos reflejados son los que describen con mayor precisión el hecho deportivo contemporáneo y que, por tanto, deben tenerse en cuenta a la hora de delimitar el modelo de deporte exigible a la universidad. Para desarrollar esta cuestión de acuerdo con el enfoque metodológico hermenéutico-crítico adoptado, damos paso al siguiente apartado en el que se ofrece una delimitación del bien interno del deporte en la universidad. De alguna manera el bien interno es el que debe ayudarnos a discriminar entre los modelos que son pertinentes y los que no.

\section{El bien interno del deporte en la universidad}

Para descubrir el tipo de práctica deportiva que corresponde ofertar a los servicios de deporte universitarios es necesario delimitar a priori su bien interno. Éste puede definirse como la meta que da sentido y legitimidad a su praxis. Por poner algunos ejemplos, en la actividad empresarial el bien interno recae en la prestación de servicios a la sociedad, en la medicina el bien interno es definido por el cuidado sanitario, y en la institución universitaria éste consiste en la educación (Cortina, 2013). Es evidente que quien se dedica a cualquiera de estos ámbitos de la práctica no puede proponerse una meta cualquiera, sino que ésta ya le 
viene dada. Así pues, el bien interno no es algo que decide una organización en particular, sino que le viene determinado y que, en consecuencia, es lo que marca necesariamente su horizonte de acción.

La delimitación del bien interno del deporte en la universidad, sin embargo, no puede apoyarse únicamente en un proceso de reflexión teórica, sino que debe tener en cuenta la opinión de quienes están involucrados directamente en su praxis. En este sentido, el artículo presenta a continuación una revisión bibliográfica y legal que recoge la idea que los principales especialistas y órganos responsables manifies$\tan$.

En principio es una ardua tarea hablar del concepto de deporte en la universidad, ya que en la actualidad es un fenómeno que aún no goza de una identidad clara y definida. En nuestro país existen diferentes concepciones, lo que provoca que en ciertos casos, como afirma LópezYeste (2009), ni siquiera quienes se encargan de su gestión lo tengan claro:

A fin de cuentas, es difícil hablar del concepto deporte universitario, por que como tal no existe. Existen universitarios que practican deporte, también existen organismos que tratan de regular el deporte para el sector de población universitaria, pero el deporte universitario en sí, no goza de un protagonismo propio que lo dote de identidad. Ni siquiera las administraciones que lo regulan saben realmente hacia donde dirigen su gestión. Se mezclan ideas, conceptos y objetivos. (p. 27)

Desvelando este mismo hecho, Aguado (2006) profundiza en la diversidad de modelos que se dan en el territorio estatal, convirtiéndose a su vez estas diferencias estructurales, en diferencias en la gestión y la organización del mismo:

En cuanto al modo en que las universidades afrontan la organización del deporte, podemos decir que no existe un modelo definido de organización deportiva universitaria, sino que conviven diferentes modelos, con diferencias, no solo en aspectos de estructuras e instalaciones sino en las diferentes políticas que las universidades llevan a cabo. (p. 155)

Dadas las dificultades para hablar de una identidad homogénea, se recorrerán a continuación las posturas más significativas con el objetivo de desvelar el auténtico bien interno de los servicios de deporte en la universidad. Para acometer dicha tarea el método hermenéutico-crítico seguido exige dar cuenta del marco deontológico, atendiendo fundamentalmente al desarrollo legal relacionado. Como es sabido, el marco legislativo es un elemento que estructura y mediatiza determinadas actividades socio-culturales, participando en ocasiones como uno de los agentes creadores de su identidad.

En el terreno del deporte en la universidad existen multitud de leyes implicadas, quedando la ordenación entre la normativa deportiva y la educativa (Hernando, 2006; Palomar, 2006). A partir de un lento y complejo proceso de configuración del deporte en launiversidad(LópezYeste, 1999), puede decirse que un primer paso significativo lo supuso la promulgación de Real Decreto 2069/1985, de 9 de octubre, sobre la articulación de competencias en materia de actividades deportivas universitarias. En él se clarifica la distribución competencial de las instituciones implicadas hasta el momento. A las universidades correspondería la organización de las actividades deportivas en su ámbito respectivo, a las comunidades autónomas la coordinación de las actividades de su territorio, y al Consejo de Universidades la coordinación general.

Dada la coexistencia de diferentes instituciones implicadas en el devenir del deporte universitario, en 1988 se publicó la Orden de 20 de diciembre por la que se creó el Comité Español del Deporte Universitario (C.E.D.U.), modificada a posteriori en la Orden 273/2004, de 3 de febrero. El C.E.D.U. es un órgano asesor y consultivo del que forman parte todas las entidades implicadas en el deporte universitario. Con posterioridad a la creación del C.E.D.U., se publicó la Ley 10/1990 del Deporte, que atribuye al Consejo Superior de Deportes y a las Comunidades Autónomas la coordinación del deporte escolar y universitario cuando éste tenga proyección nacional e internacional.

En cuanto a las leyes de deporte autonómicas, destacar que éstas no llegan mucho más lejos al definir el deporte en la universidad. Hernando (2006) señala que son varias las que se suscriben a la siguiente definición recogida en el Artículo 57 de la ley del deporte del País Vasco: «Toda actividad deportiva, competitiva orecreativa practicada exclusivamente por la población universitaria en el seno de los programas deportivos de las universidades». Definición que, según se adelantaba, se refleja en la misma línea en la ley del deporte de la Comunidad de Murcia, la de Navarra y la de Castilla y León.

En relación a la legislación educativa destaca el papel de la L.O.M.L.O.U., en su Artículo 90, donde expone que el deporte en la universidad tiene el sentido de contribuir no sólo a la mejora de la salud, la calidad de vida y las condiciones físicas, sino también a la formación en valores y competencias, contribuyendo a la formación integral.

Por otra parte, el Real Decreto 1791/2010, de 30 de diciembre, recoge el Estatuto del Estudiante Universitario. En su artículo 62.1 señala que las actividades deportivas de los estudiantes universitarios podrán orientarse hacia la práctica de deportes y actividades deportivas no competitivas o hacia aquellas organizadas en competiciones internas, autonómicas, nacionales o internacionales. Cosa que deja el camino abierto a dos de los tres modelos deportivos analizados previamente: deporte-praxis y deporte de competición.

Más allá del desarrollo legal, el marco deontológico también lo compone la voz de los expertos y protagonistas de la praxis en cuestión. En este sentido, los foros especializados defienden que el modelo deportivo universitario debe contar con un carácter diferenciador y formativo que lo defina y que lo dote de una identidad propia y alineada con la misión de la universidad (López-Yeste, 2002). Idea que constata Canibe (2011) al destacar la necesidad de entender que el deporte en la universidad no es, o al menos no debe ser, únicamente el deporte que se practica dentro de la universidad por el mero hecho de desarrollarse en dicho emplazamiento, sino que necesita tener un carácter diferenciador centrado en su capacidad formativa. Un carácter basado, según este autor, en el desarrollo de valores, la formación integral y toda una serie de componentes que le permitan desplegar una identidad propia.

Por suparte, Hernando (2010) reafirma lo dicho indicando que: «La actividad física que se realice en las universidades debe contribuir a la formación integral del estudiante, permitir el crecimiento personal y generar hábitos que ayuden a la mejora de la calidad de vida y contribuyan al bienestar de las personas» (p. 56).

Del mismo modo, Almorza, Yébenes, Rivas y Bablé (2010) concluyen que los servicios de deporte deben fundamentar su programación según el principio de:

Contribuir a la formación integral de los alumnos a través del deporte, que es el mandato imperativo de las universidades a sus Unidades de Deportes: ir más allá de la simple programación de ocio y complementar la misión educativa encomendada por la sociedad a la universidad. (p. 201)

Finalmente, en la misma línea argumentativa Almorza et al.(2011) refrendan lo anterior cuando responden a:

¿De qué deporte estamos hablando cuando nos referimos a este $<<$ Deporte Universitario $>>$ ? Indiscutiblemente, al que tiene como objetivos la mejora de la salud, de la condición física y de la calidad de vida; conseguir un mayor desarrollo de las cualidades físicas básicas y habilidades motrices; convertirse en una alternativa de ocupación del tiempo de ocio; ser un vehículo para el establecimiento de relaciones sociales...Pero sobre todo, y éste es el elemento diferenciador fundamental, el que tiene como premisa imprescindible $<<$ Contribuir a la formación integral de los alumnos, a su formación en valores y a la adquisición de competencias >>. (p. 39)

De hecho, ¿qué otro sentido tendría la práctica de deporte en el seno de una institución educativa como la universidad sino su contribución a la formación integral? En esta línea, Martínez (2006) advierte que la universidad ha estado volcada en la actividad intelectual, no sabiendo reconocer la contribución que el deporte puede tener en el desarrollo armónico de su población. Además, en palabras de Morales (2009) diremosque:

Ahí es donde desde nuestro planteamiento creemos que debe encuadrarse el deporte en la universidad, como complemento a la docencia y a la investigación que favorezca, entre otros, la dimensión de los 
valores que la propia Universidad busca, potenciando su implicación social, gracias a sus aspectos dinamizadores. (p. 86)

En efecto, el carácter diferenciador basado en la formación integral es uno de los aspectos a destacar en la definición que se persigue del deporte en la universidad. No obstante, la cosa no queda aquí, sino que llegando un poco más lejos, veremos a continuación si el deporte en la universidad debe cumplir su función formadora únicamente con el alumnado, o si por el contrario, debería abrirse a toda la población universitaria. No en vano, Almorza et al. (2011) indican que casi una sexta parte de la comunidad universitaria es usuaria de los servicios de deportes, siendo cerca del $12 \%$ usuarios de los colectivos de Personal de Administración y Servicios (P.A.S), y Personal Docente e Investigador (P.D.I.).

Sin embargo, aún desde esta concepción del deporte en la universidad como deporte abierto a toda la comunidad universitaria, éste no debe interpretarse como el deporte de empresa con fines higiénicorecreativos sino que debe trascender, desde sus posibilidades formadoras, incluso hacia aquellos colectivos que, aún siendo cercanos, no pertenecen directamente a la comunidad universitaria. Hecho que obliga a reflexionar sobre la función del deporte en la universidad también desde su vertiente de extensión universitaria. Es decir, ¿¿debe la universidad, en su función social y de extensión, fomentar y promover su modelo deportivo de un modo democratizador hacia todo su entorno social? Nuestra opinión es que sí. Aunque damos paso a otras voces autorizadas para apoyar nuestra postura.

Roca (2006) indica que al abordar la gestión de los servicios de deporte universitarios conviene tener claro que éstos no sólo han de responder ante los estudiantes o colectivos implicados laboralmente en el funcionamiento de la universidad, sino que deben ampliar el radio de acción al entorno social universitario. Es decir, a los grupos sociales que mantienen relaciones de interacción con la institución universitaria. Éste hecho también ha sido puesto sobre la mesa por Morales (2009), quien indica la existencia de este nuevo foco de acción:

Pues bien, esa doble perspectiva se refiere al enfoque interno como principal referencia de actuación, la comunidad universitaria y otro al enfoque de apertura, donde además de ésta participan otras personas que toman parte de la vida social de la universidad y su entorno, pasando a ser un objetivo social cada vez más significativo. (p. 85)

Así pues, a la vista de este nuevo objetivo social, a los servicios de deporte universitarios corresponderá articular los medios para tratar de garantizar en lo posible dicha práctica deportiva no sólo al alumnado o a la comunidad universitaria, sino también al resto de la sociedad. Para rematar con claridad este posicionamiento, nos apoyamos en Morales (2009) cuando dice que:

Denominamos Comunidad Universitaria al conjunto de población universitaria que está compuesta por el personal docente e investigador, estudiantes y el personal de administración y servicios, y aunque todas las estructuras organizativas del deporte universitario tienen claro que el foco principal de atención lo configuran los estudiantes de la Universidad, las propuestas deben hacerse extensivas al resto de los diversos colectivos tanto de la comunidad universitaria como del entorno social, teniendo en consideración los diferentes objetivos que persiguen cada uno de éstos, planteando en algunos casos alguna oferta específica que responda a unas peculiaridades muy concretas. (p. 86)

En definitiva, de lo dicho hasta el momento debe quedar la idea de que el bien interno del deporte en la universidad radica en promover un enfoque educativo del deporte en la universidad que impregne las propuestas de los servicios de deporte universitarios. Pero además, no debe olvidarse un importante enfoque social que permita expandir su radio de acción más allá del entorno universitario, siendo todo ello gestionado desde una perspectiva integradora a partir de su alineación con las legítimas metas de la institución universitaria. Por tanto, el modelo de deporte en la universidad que cabe defender emerge de aquellas vertientes del hecho deportivo descritas en el apartado anterior que concuerden con el bien interno definido. Este análisis queda desarrollado detenidamente en el siguiente apartado.

\section{Descripción del modelo español de deporte en la universidad}

Una vez delimitado el bien interno que da sentido a los servicios de deporte en la universidad, el presente apartado analiza el estado de la cuestión en España. El propósito de este punto se centra en explicitar el modelo de deporte en la universidad actual, para así justificar de un modo realista y apropiado las orientaciones de gestión que ofrecemos en última instancia.

A la hora de describir un modelo de deporte en la universidad, el que sea, existe una variedad tan grande de criterios y enfoques posibles que el Consejo de Europa (1999) defiende el uso de tres indicadores para aclarar la cuestión y poder establecer comparativas: la filosofía del deporte, la intervención legislativa y la intervención financiera.

En el análisis desarrollado hasta el momento se han abordado dos de esos tres ejes. Tanto la filosofía del deporte como la intervención legislativa han sido tratadas anteriormente, por lo que únicamente rescataremos en este punto los elementos más destacados. Asimismo queremos resaltar que a la intervención legislativa corresponde convertir en derecho positivo las demandas sociales legítimas, luego el modelo a seguirno debe interpretarse necesariamente a partir de las leyes vigentes, sino que son las leyes las que deben aproximarse al modelo exigible para definirlo en términos jurídicos.

Finalmente, en cuanto al tema de la intervención financiera, destacar que el grueso del sustento económico de los servicios de deporte universitarios españoles corre por parte del presupuesto de cada universidad (Almorza, et al., 2011). Anivel financiero, el nuestro es un modelo que no depende estructuralmente de sistemas privados de financiación basados en donaciones o explotación económica de los eventos efectuados.

En cualquier caso, la descripción que aquí pretendemos desarrollar aspira a profundizar un poco más, por lo que basaremos el análisis en desvelar qué vertientes del hecho deportivo contemporáneo tienen razón de ser en el modelo de deporte en la universidad. Pues, como se ha visto, la configuración del deporte en la universidad depende de un número de variables bastante amplio que cabe acotar con mayor detalle.

De esta manera, una vez descrita la situación actual del hecho deportivo contemporáneo y delimitado el bien interno del deporte en la universidad, estamos en disposición de definir qué modelos o vertientes del hecho deportivo son susceptibles de ser incorporados en la oferta de los servicios de deporte universitarios. A priori, según los principios identitarios del deporte en la universidad recién desvelados, el modelo de deporte que correspondería promover a la institución universitaria debe integrar elementos tanto del denominado deporte para todos o deporte praxis, como del deporte de competición o de rendimiento, quedando completamente al margen el deporte espectáculo.

En la figura 1 se muestra cómo queda el reflejo de las diferentes clasificaciones del hecho deportivo contemporáneo en el modelo deportivo universitario propuesto.

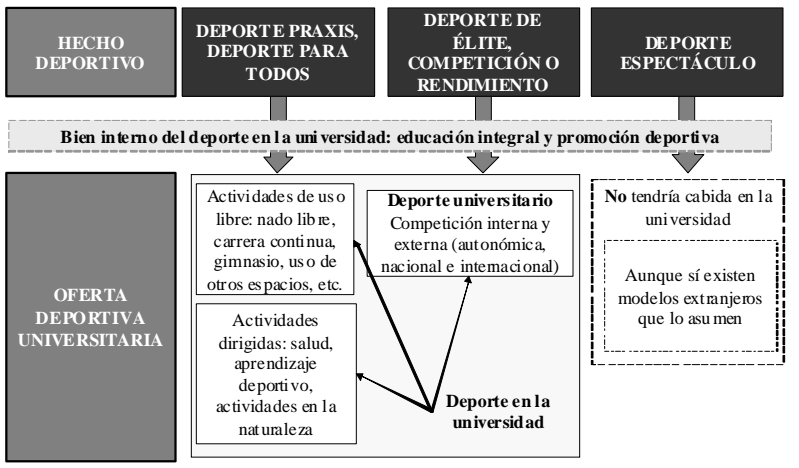

Figura 1. Reflejo del hecho deportivo en la ofert a deportiva un iversitaria. (Elaboración propia)

Como se ve, del deporte praxis o deporte para todos surge una oferta centrada en actividades dirigidas y actividades de uso libre, mientras que del deporte de competición o deporte rendimiento emerge la parte relativa a la competición universitaria. 
Este planteamiento alejado del deporte espectáculo coincide con el modelo planteado por el Consejo Superior de Deportes (2010). En él pueden observarse con más detalle cuales son las especificidades de las distintas propuestas: actividades dirigidas, actividades de uso libre y competición universitaria.

Relativo a las actividades dirigidas, éstas pueden a su vez clasificarse en tres bloques. El primero relativo a las actividades orientadas a la salud, el segundo en conexión con las actividades de aprendizaje deportivo y el tercero relacionado con las actividades en la naturaleza.

Por su parte, las actividades de uso libre reaccionan a la gran demanda existente, generando programas que permiten un fácil acceso a las instalaciones deportivas. Algunos ejemplos habituales se encuentran en la práctica de natación o actividades acuáticas sin monitor, la carrera continua, el uso de gimnasios, etc.

En cuanto a la competición universitaria, Hernando (2010) plantea la necesidad de que se reconozca como un factor esencial del entramado deportivo universitario. En este punto conviene matizar que es imprescindible enfocarla como una finalidad educativa y formadora, en tanto que acarrea elementos como el afán de superación, el sacrificio, el valor del esfuerzo, la constancia, la colaboración, el respeto por el contrario y las normas, la capacidad de liderazgo, el trabajo en equipo, la autocrítica, etc. Tras esta reflexión previa, decir que las universidades articulan sus competiciones en dos niveles: la competición interna, con un carácter predominantemente participativo y social; y la competición externa (nacional e internacional), centrada en la representación universitaria. Para acabar de precisar cómo queda configurado el modelo español de deporte en la universidad, conviene hacer una última aclaración. Como se ha visto, dentro del modelo deportivo universitario español existen dos formas de entender la práctica bien diferenciadas. Por una parte existe lo que, desde el deporte praxis o deporte para todos, algunos apuntan como deporte en la universidad; mientras que, por otra parte, existe una concepción del deporte de carácter más competitivo conocida como deporte universitario (Hernando, 2010; López-Yeste 1999). El uso de una u otra expresión denota un posicionamiento y un significado inequívoco entre los especialistas y profesionales del área. Por lo que ha de quedar claro que deporte en la universidad y deporte universitario no son términos sinónimos cuando provienen de foros especializados.

Como deporte en la universidad se entiende entonces al conjunto de la práctica físico-deportiva realizada no sólo por los estudiantes universitarios sino también por el resto de la comunidad universitaria (colectivo profesional) y ciudadanos practicantes dentro de la oferta de la universidad. Ello permite entender que se trata de una oferta que atañe a un colectivo muy amplio cuya formación físico-deportiva no finaliza con los estudios de grado, sino que se sigue desarrollando independientemente de la edad y el enfoque de práctica que se elija. El deporte en la universidad engloba una formación integral durante toda la vida. Además, no conviene olvidar que éstos desempeñan un papel vital en la función de extensión universitaria, puesto que ejercen como agentes de extensión de los conocimientos, experiencias y valores de la universidad hacia el resto de la comunidad.

Por otra parte, lo que se entiende como deporte universitario pertenece a una idea relacionada con el deporte de competición que practican exclusivamente los estudiantes universitarios en edades comprendidas entre los 18 y los 28 años (rango de edad para participar en las actividades de la Federación Internacional de Deporte Universitario). En cualquier caso no se trata de concepciones excluyentes, pues es de recibo admitir que el deporte universitario no es más que una de las formas de práctica físico-deportiva que se lleva a cabo dentro de la concepción más global de deporte en la universidad.

Orientaciones para la gestión ética de los servicios de deporte universitarios

Una vez fundamentado y delimitado el modelo de deporte universitario, el siguiente paso es integrar la concepción alcanzada en el modelo de gestión de los servicios de deporte universitarios. En primer lugar se insta desde aquí a seguir una serie de orientaciones basadas en los planteamientos del Informe del Deporte en la Universidad, del Plan Integral para la Actividad Física y el Deporte (Consejo Superior de Deportes, 2010). Este informe plasma una serie de medidas, acciones y proyectos que determinan de un modo concreto el horizonte de gestión que los propios especialistas marcan al deporte en la universidad como institución social.

De los cuatro núcleos de contenido que configuran el informe, se desprende un especial interés por aspectos como el deber de los servicios de ofrecer un modelo de deporte que se alinee con la formación integral y continua, así como con la mejora de la salud. También destaca el interés por la formación continua, tanto de los practicantes como de los profesionales. Asimismo, se demanda impulsar la investigación sobre el propio campo deportivo en pos de transferir en lo posible los resultados obtenidos a la gestión. Finalmente, se insta a promover un progresivo acercamiento entre la universidad y la sociedad, dando una especial atención a los colectivos en mayor riesgo de exclusión social.

Por otra parte, más allá de la visión institucional que plantea el Consejo Superior de Deportes (2010), desde aquí planteamos nuevas propuestas entorno a dos ejes indisolubles sobre los que apoyar la gestión diaria de los propios servicios de deporte: la gestión estratégica y la ética.

A nivel estratégico la gestión de los servicios de deporte universitarios implica una serie de tareas relacionadas con:

- La gestión económica. Los recursos económicos deben contenerse en los tiempos de crisis que corren. Decir lo contrario no sería realista ya que las universidades están sufriendo una serie de medidas de ajuste que no permiten otra opción. En cualquier caso, sería deseable la determinación de un porcentaje presupuestario mínimo por parte de las universidades, así como estudiar fórmulas que, alineadas con la función de extensión universitaria, rentabilizaran la apertura de los servicios de deporte a la sociedad.

Ø La gestión de instalaciones y programas de actividad física. La construcción, mejora y mantenimiento de nuevas instalaciones debe seguir progresando. Principalmente en términos de cuidado y eficiencia. En cuanto al diseño de programas de actividad física, se insta a seguir trabajos en la línea de los presentados por Castañeda y Campos (2012), Flores y Ruiz (2010) y Zagalaz, Lara y Cachón (2009), en los que se analizan las motivaciones de estudiantes de diferentes universidades hacia la práctica de actividad físico-deportiva, destacando entre otros parámetros e intereses: la salud, las relaciones sociales, la adecuación a su nivel de práctica, la disponibilidad horaria, etc. Asimismo, de acuerdo con Consejo Superior de Deportes (2010), conviene repensar nuevas fórmulas de explotación de espacios y servicios para promocionar la accesibilidad de colectivos en riesgo de exclusión social.

Ø La gestión de los recursos humanos. Ésta es una tarea vital para el buen funcionamiento de los servicios. En este sentido, se sugiere un modelo de gestión que responda a los intereses legítimos de los trabajadores, creando espacios de diálogo y herramientas centradas en facilitar la interacción.

$\varnothing$ La gestión de los servicios con parámetros de calidad. Es decir, debe prevalecer la vocación de servicio y, por tanto, una apuesta seria y auditable para incrementar la calidad del servicio prestado. En esta línea, se propone incorporar la reciente Norma I.S.O. 26000, diseñada como Guía de Responsabilidad Social.

En plena sintonía con estos puntos estratégicos debe emerger, necesariamente, un modelo de gestión ética que propicie una guía de actuación capaz de alinear la gestión desarrollada con el bien interno y la responsabilidad de los servicios de deporte universitarios.

A lo largo del artículo se han identificado muchos intereses que desde diferentes grupos de implicados constituyen la idiosincrasia del deporte en la universidad y que, por tanto, necesitan ser integrados en su gestión. Respondiendo a esta concepción plural se propone en este punto importar, desde la teoría general de gestión empresarial, el modelo de gestión de stakeholders planteado por Freeman (1984).

El concepto stakeholder se define como grupo de interés que afecta y/o puede ser afectado por la toma de decisiones de la entidad. En el 
marco de los servicios de deporte en la universidad, algunos de los stakeholders más significados serían los usuarios, los trabajadores, la propia administración universitaria, los proveedores de productos y servicios, otras universidades y administraciones públicas, etc.

Este planteamiento exige una relación bidireccional constante entre la entidad y los grupos de interés, ya que ésta se concibe pluralmente a partir de los mismos. Así, se integran los inputs o factores externos en los procesos de toma de decisiones, pensando en las consecuencias y los efectos de las acciones sobre los stakeholders.

\section{Conclusiones}

Este artículo constata que el deporte en la universidad es un fenómeno complejo que en las últimas décadas ha experimentado un importante auge. En este sentido, existen una serie de indicadores como el número de participantes y personas implicadas, el volumen de dinero invertido, el potencial educativo y saludable de la práctica deportiva, la diversidad de modelos de práctica, etc., que hacen exigible un serio proceso de reflexión sobre el que fundamentar y orientar su gestión futura.

Esta necesidad de reflexión y deliberación exige que, más allá de analizar datos y tendencias sobre cómo es del deporte en la universidad, se esgriman también argumentos y criterios sólidos, racionales y filosóficamente fundamentados, sobre cómo debe ser. Esta iniciativa se ha llevado a cabo a partir de una aproximación metodológica hermenéuticocrítica, centrada en desvelar desde el interior de la propia praxis el bien interno que la legitima y da sentido, para proponer a partir de dicho análisis los medios y procedimientos más adecuados para guiar su gestión.

Este camino no se ha recorrido sin dificultad. Uno de los hándicaps más significativos ha sido el problema de definir el propio modelo español de deporte en la universidad. No obstante, a partir de los diferentes modelos contemporáneos de deporte identificados, se ha concluido que el deporte en la universidad debe configurarse a partir de la promoción y desarrollo tanto del modelo de deporte-praxis como del de deporte de competición, acentuando el sentido educativo y saludable de ambos.

A partir de dicho análisis el artículo ha planteado una serie de propuestas de mejora tanto a nivel institucional, tomando como punto de partida en el Informe de Deporte en la Universidad del Plan Integral para la Actividad Física y el Deporte (Consejo Superior de Deportes, 2010); como a nivel organizacional, centradas en la mejora de la gestión estratégica y ética de los propios servicios de deporte universitarios.

A nivel de gestión estratégica se han propuesto una serie de ejes como: la necesidad de optimizar y ajustar la gestión económica a los tiempos que corren; el desarrollo de nuevas fórmulas de explotación de espacios y servicios; la necesaria gestión de recursos humanos de acuerdo con la satisfacción de los intereses legítimos de los trabajadores; y, finalmente, la puesta en valor de los diferentes parámetros de calidad aplicados a los procesos de gestión.

En conexión con estas sugerencias estratégicas, a nivel de gestión ética se ha propuesto, como paradigma de gestión, el modelo de stakeholders planteado por Freeman (1984). Este modelo, importado desde la teoría general de gestión empresarial, plantea dar voz a los diferentes grupos de implicados, de manera que se conozcan y sean tenidos en cuenta los intereses de los mismos a la hora de tomar decisiones.

\section{Referencias}

Aguado, J. L. (2006). Estructura del deporte universitario. En R. Teruel (Dir.), E deporte universitario en España: actualidad y perspectivas de futuro (pp.151167). Madrid: Dykinson.

Almeida, M. (2001). El papel de la universidad contra la barbarie. Revista De Estudios Orteguianos, 2, 111-118.

Almorza, D., Yébenes, A., Rivas, R., \& Bablé, J. A. (2010). El deporte universitario en Andalucía. Cádiz: Servicio de publicaciones de la Universidad de Cádiz.

Almorza, D., Yébenes, A., Bablé, J. A., Rivas, R., Ronquete, J., \& Casadi, I. (2011). Estudio Diagnóstico del Deporte Universitario Español. Cádiz: Servicio de publicaciones de la Universidad de Cádiz.
Cagigal, J. M. (1996). Obras selectas. Madrid: C.O.I., A.E.D.P., Ente de promoción deportiva J.M. Cagigal.

Canibe, A. (2011). El papel del deporte en los campus de excelencia universitarios. Tándem, 38-46.

Castañeda, C., \& Campos, M. C. (2012). Motivación de los estudiantes de la Facultad de Ciencias de la Educación (Universidad de Sevilla) hacia la práctica de actividad físico-deportiva. RETOS, Nuevas tendencias en Educación Física, Deporte y Recreación, 22, 57-61.

Conill, J. (2006). Ética Hermenéutica. Crítica desde la facticidad. Madrid: Tecnos. Consejo de Europa. (1999). Informe de la Comisión al Consejo Europeo. COM 644.

Consejo Superior de Deportes. (2010). Plan integral para la actividad física y el deporte. [Versión electrónica]. Recuperado el 11 de Febrero de 2014 de: http:/ /www.csd.gob.es/csd/sociedad/plan-integral-para-la-actividad-fisica-y-el-deporteplan-a-d/plan-integral-para-la-actividad-fisica-y-el-deporte/

Cortina, A. (1993). Ética aplicada y democracia radical. Madrid: Tecnos.

Cortina, A. (2013). ¿Para qué sirve realmente...? La ética. Barcelona: Paidós.

Domínguez, J. L. (1995). Reflexiones acerca de la evolución del hecho deportivo. Bilbao: Universidad del País Vasco.

Elias, N., \& Dunning, E. (1986). Quest for Excitement: Sport and Leisure in the Civilising Process. Londres: Blackwell.

Flores, G., \& Ruiz, F. (2010). Motivaciones de los estudiantes universitarios para nunca realizar actividades físico-deportivas de tiempo libre. El caso de la Universidad de Guadalajara. RETOS. Nuevas tendencias en Educación Física, Deporte y Recreación, 17, 34-37

Freeman, R. E. (1984). Strategic management. A Stakeholder Aproach. Toronto: Pitman.

García Ferrando, M. (1990). Aspectos sociales del deporte. Una reflexión sociológica. Madrid: Alianza Editorial.

García Ferrando, M. (2006). Postmodernidad y deporte: encuesta sobre hábitos deportivos de los españoles 2005. Madrid: Centro de Investigaciones Sociológicas.

García Ferrando, M., \& Llopis, R. (2011). Ideal democrático y bienestar personal: los hábitos deportivos en España 2010. Madrid: Ministerio de Educación y Ciencia.

Hernando, C. (2006). Comunidades Autónomas y Deporte Universitario. En R. Terol, (Dir.), El deporte universitario en España: actualidad y perspectives de futuro (pp. 207-226). Madrid: Dykinson.

Hernando, C. (2010). El Plan Integral para la Actividad Física y el Deporte. Ámbito de la Actividad Física y Deporte en la Universidad. Tandem, Monográfico Deporte y Universidad, 35, 25-37.

Isidori, E. (2011). La pedagogía del deporte como ciencia. Perspectivas críticas. En Isidori, E. \& Fraile, A. La pedagogía del deporte hoy. Horizontes y desafíos. (pp. 1- 42). Roma: Edizioni Nuova Cultura.

López Yeste, A. (1999). El deporte en la Universidad Politécnica de Valencia. Un estudio desde la psicología social del consumidor. Tesis doctoral. Universidad de Valencia, Valencia, España.

López Yeste, A. (2002). Gestión del Deporte Universitario. Actas del I Congreso de Gestión del Deporte de la Asociación de Gestores profesionales de la Comunidad Valenciana. (pp. 37-45), Valencia, España.

López Yeste, A. (2009). IV Congreso de Gestión del Deporte. Valencia: Servicio de publicaciones de la Universidad Politécnica de Valencia.

Martínez, V. (2006). Orientación y tendencias del deporte universitario en España. En R. Terol (Dir.), El deporte universitario en España: actualidad y perspectivas de futuro (173-184). Madrid: Dykinson.

Mechikoff, R. A., \& Estes, S.G. (2005). A history and philosophy of sport and physical education. From ancient civilizations to the modern world. New York: Mc Graw Hill.

Miller, T., Lawrence, G. A., McKay, J., \& Rowe, D. (2001). Globalization and sport. London: Sage publications Ltd.

Morales, M. A. (2009). La organización y gestión de las actividades físicodeportivas en la Universidad: un caso práctico. Tesis Doctoral. Universidad de Málaga. Málaga, España.

Palomar, A. (2006). El deporte universitario en el sistema deportivo. En R. Terue (Dir.), El deporte universitario en España: actualidad y perspectivas de futuro (pp. 35-70). Madrid: Dykinson.

Parlebás, P. (2001). Juegos, deporte y sociedad. Léxico de praxiología motriz. Barcelona: Paidotribo.

Puig, N., \& Monteagudo, M. J. (2004). (Eds.). Ocio y deporte. Un análisis multidisciplinar. Bilbao: Universidad de Deusto.

Roca, J. (2006). El deporte universitario en el ámbito andaluz. En R. Teruel (Dir.), El deporte universitario en España: actualidad y perspectivas de futuro (pp. 185-202). Madrid: Dykinson.

Rodríguez Díaz, A. (2008). El deporte en la construcción del espacio social. Madrid: Centro de Investigaciones Sociológicas.

Vicente, M. (2011). De la diversidad del concepto de deporte y su naturaleza. En E. Isidori \& A. Fraile (Coord.), La pedagogía del deporte hoy. Escenarios y desafíos (pp. 43-80). Roma: Edizioni Nuova Cultura.

Zagalaz, M. L., Lara, A. J., \& Cachón, J. (2009). Utilización del tiempo libre por el alumnado de magisterio de la especialidad de educación física. Análisis en la Universidad de Jaén. RETOS, Nuevas tendencias en Educación Física, Deporte y Recreación, 16, 104-107. 